Peter Czimmermann - Michal Kohani*

\title{
CHARACTERISTICS OF CHANGES OF TRANSPORTATION PERFORMANCE FOR PAIRS OF CRITICAL EDGES
}

When the robustness of a public service system design is tested, we can often use scenarios where possible random failures can occur and they can influence the time the service is accessible which is provided for system users. The construction of a suitable scenario is based on the choice of links of the transportation network which influence the system performance in a substantial way. In such scenarios one or multiple arcs can be affected by this failure. In our contribution we present characteristics of pairs of critical arcs that can be used to develop an algorithm for the creation of critical scenarios.

Keywords: critical scenario, system robustness, transportation performance, public service system

\section{Introduction}

The characteristics of public service systems in transportation networks are given by the location of its service centres. Examples of such systems include emergency, health care and supply systems. The service for users is realized on the shortest paths between the users and service centres. A design of such a system can be solved as a weighted $p$-median problem in a network. The algorithms and methods for solving these types of problems are known and can be found in [1], [2], [3].

However, the service systems are usually suggested for ideal conditions, when the traverse time is constant for every edge. In real transportation networks, various random events may occur. These events can elongate the traverse time of the affected edges (arcs) [4]. The collapse of an individual arc was studied in [5], [6], [7]. Two characteristic functions were suggested for measuring the system robustness regarding possible disruptive events on network arcs. The network robustness index was studied in [6]. The network trip robustness was suggested in [7]. Characteristic function of individual network arcs was also studied in [8].

In this paper, we focus on the characterization of pairs of affected edges. We suggest an approach that allows us to compute the function of the elongation of transportation performance when two edges are affected by random events at the same time.

\section{Definition of transportation performance}

In this section we introduce basic definitions. We work with the network $G=(V, E, w, t)$, where $V$ is the set of vertices, $E$ is the set of edges, $w(u)$ is the weight of the vertex $u$, and $t(e)$ is the driving time through the edge $e$. The driving time from vertex $u$ to vertex $v$ is denoted by $d(u, v)$ and it is the length of the shortest path from $u$ to $v$ when we consider the times on the edges from $E$. Similarly, we can define the value

$d(u, X)=\min \{d(u, v) ; v \in X\}$ for every non-empty subset of vertices $X \subset V$.

Let the set of customers be denoted by $U$ and the set of facilities be denoted by $S$, where $U \subset V, S \subset V$. The transportation performance [8] is

$W=\sum_{u \in U} w(u) \cdot d(u, S)$

This expression is similar to the total weighted distance, however we use travel times on edges, instead of distances.

\section{Changes of the transportation performance}

It is not hard to imagine situations when the travel time on the edge is extended. In this section we describe the impact of such extensions on transportation performance. We focus on the case with two critical edges.

Let the set

$Y=\left\{e_{1}, e_{2}, \ldots, e_{k}\right\} \subseteq E$

be given. We suppose that $\delta_{i} \geq 0(i=1,2, \ldots, k)$ is an extension of the driving on the edge $e_{i}$. We denote the transportation performance with extended times

$t\left(e_{i}\right)+\delta_{1}, t\left(e_{2}\right)+\delta_{2}, \ldots, t\left(e_{k}\right)+\delta_{k}$

on edges of $Y$ by

$W_{Y}\left(\delta_{1}, \delta_{2}, \ldots, \delta_{k}\right)$

We can see that $W_{Y}$ is a function of $k$ variables $\delta_{1}, \delta_{2}, \ldots, \delta_{k}$ with domain $R_{+}^{k}$.

The case $k=1$ is studied in [8]. We are concerned with the case $k=2$ and consider some possibilities for $k>2$.

\footnotetext{
* Peter Czimmermann, Michal Kohani

Department of Mathematical Methods and Operations Research, Faculty of Management Science and Informatics, University of Zilina, Slovakia E-mail: peter.czimmermann@fri.uniza.sk
} 


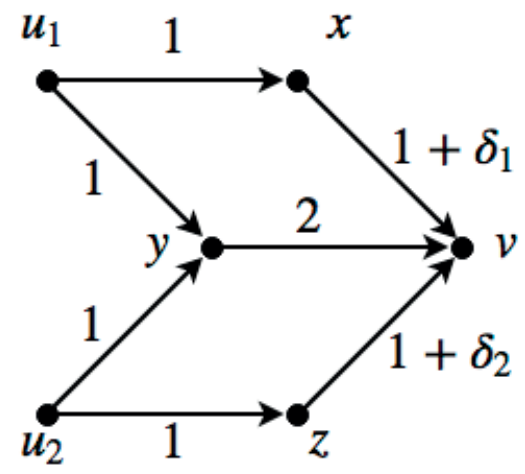

Figure 1 Network from Example 1

Example 1. We consider the network $G$ in Figure 1. Let $U=\left\{u_{1}, u_{2}\right\}$ and $S=\{v\}$. Transportation performance $W_{Y}\left(\delta_{1}, \delta_{2}\right)$ is given by formulas:

1. $4+\delta_{1}+\delta_{2}$, if $0 \leq \delta_{1}, \delta_{2} \leq 1$

2. $5+\delta_{1}$, if $0 \leq \delta_{1}$ and $\delta_{2}>1$

3. $5+\delta_{2}$, if $0 \leq \delta_{2}$ and $\delta_{1}>1$

4. 6 otherwise

We can see that the following facts hold for every network and pair of its edges.

Theorem 1. For the given vertex $u$, the transportation performance is given by, at most, four formulas.

Proof. There are four cases to consider:

Case $c_{3}$ - the edges $e_{1}, e_{2}$ belong to the shortest path from $u$ to $S$ for given values $\delta_{1}, \delta_{2}$.

Case $c_{1}$ - the edge $e_{1}$ belongs ( $e_{2}$ does not) to the shortest path from $u$ to $S$ for given values $\delta_{1}, \delta_{2}$.

Case $c_{2}$ - the edge $e_{2}$ belongs ( $e_{1}$ does not) to the shortest path from $u$ to $S$ for given values $\delta_{1}, \delta_{2}$.

Case $c_{0}$ - the edges $e_{1}, e_{2}$ do not belong to the shortest path from $u$ to $S$ for given values $\delta_{1}, \delta_{2}$.

The number of formulas is equal to the number of cases $c_{3}, c_{1}, c_{2}$, $c_{0}$ that can occur. We denote the sets of points $\left(\delta_{1}, \delta_{2}\right)$, for which cases $c_{3}, c_{1}, c_{2}, c_{0}$ hold, by $\Delta_{3}, \Delta_{1}, \Delta_{2}, \Delta_{0}$. We can compare these sets by the following relation:

$\Delta_{i} \rightarrow \Delta_{j} \Leftrightarrow$ there exists point $(a, b) \in \Delta_{i}$ such that for all $(c, d) \in \Delta_{j}$ we have $a \leq c, b \leq d$ and at least one inequality is sharp. It is possible to show by case by case analysis that the relationships between the sets $\Delta_{3}, \Delta_{1}, \Delta_{2}, \Delta_{0}$ are in Figure 2. It is easy to consider that every case is represented by one formula (we have one formula for every set), as we can see below:

- case $c_{3}: w(u) \cdot\left(d_{3}(u, S)+\delta_{1}+\delta_{2}\right)$, where $d_{3}(u, S)$ denotes the length of the shortest path from $u$ to $S$, when $\left(\delta_{1}, \delta_{2}\right) \in \Delta_{3}$, (this path is the shortest among all paths from $u$ to $S$, which contain the edges $e_{1}$ and $e_{2}$ )

- case $c_{1}: w(u) \cdot\left(d_{1}(u, S)+\delta_{1}\right)$, where $d_{1}(u, S)$ denotes the length of the shortest path from $u$ to $S$, when $\left(\delta_{1}, \delta_{2}\right) \in \Delta_{1}$,

- case $c_{2}: w(u) \cdot\left(d_{2}(u, S)+\delta_{2}\right)$, where $d_{2}(u, S)$ denotes the length of the shortest path from $u$ to $S$, when $\left(\delta_{1}, \delta_{2}\right) \in \Delta_{2}$,

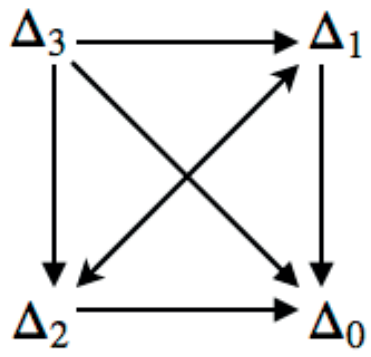

Figure 2 Comparison of sets $\Delta_{3}, \Delta_{1}, \Delta_{2}, \Delta_{0}$

- case $c_{0}: w(u) \cdot d_{0}(u, S)$, where $d_{0}(u, S)$ denotes the length of the shortest path from $u$ to $S$, when $\left(\delta_{1}, \delta_{2}\right) \in \Delta_{0}$.

Theorem 2. The function $W_{Y}\left(\delta_{1}, \delta_{2}\right)$ is continuous and piecewise linear function in two variables $\delta_{1}, \delta_{2}$.

Proof. Let the vertex $u \in U$ be given. Let the edges $e_{1}, e_{2}$ belong to the shortest path from $u$ to $S$. If the travel times on edges $e_{1}, e_{2}$ are increased by values $\delta_{1}, \delta_{2}$, then the travel time $d(u, S)$ from $u$ to $S$ is changed to $d(u, S)+\delta_{1}+\delta_{2}$. If the value of $\delta_{2}$ achieves the level which involves omitting the edge $e_{2}$, then we obtain a new formula for the travel time from $u$ to $S: d_{1}(u, S)+\delta_{1}$. Moreover, the transition between these formulas is given by equality:

$d(u, S)+\delta_{1}+\delta_{2}=d_{1}(u, S)+\delta_{1}$

It follows from the equality that this transition is continuous. The same can be checked for all cases. The general formula for the transportation performance is

$$
W_{Y}\left(\delta_{1}, \delta_{2}\right)=\sum_{u \in U} w(u) \cdot\left(d_{i}(u, S)+a_{i} \cdot \delta_{1}+b_{i} \cdot \delta_{2}\right)
$$

where $i \in\{0,1,2,3\}$; its value depends on $\left(\delta_{1}, \delta_{2}\right) \in \Delta_{i}$ and $a_{0}=b_{0}=0, a_{1}=1, b_{1}=0, a_{2}=0, b_{2}=1, a_{3}=b_{3}=1$. It is known that the sum of continuous functions is a continuous function and from the general formula it follows that $W_{Y}\left(\delta_{1}, \delta_{2}\right)$ is piecewise linear function.

\subsection{Computation of transportation performance for a given vertex $u$}

It was mentioned in the proof of Theorem 2 that the general formula for $W_{\gamma}\left(\delta_{1}, \delta_{2}\right)$ is the sum of formulas derived for each vertex from the set $U$. Hence, we show the approach for the computation of formulas for every vertex from this set. We also show the computation of boundaries of sets with the same formula. Composition of these sets provides the partition of the domain of $W_{Y}\left(\delta_{1}, \delta_{2}\right)$. We show the approach which gives the formula for transportation performance for a given vertex $u \in U$. 1. We find the shortest path from $u$ to $S$ in $G$ (we use values $\left.\delta_{1}=\delta_{2}=0\right)$. The length of this path is $d_{i}(u, S)$. Now, when we carefully increase the values of $\delta_{1}, \delta_{2}$ (the word carefully means that the shortest path is not changed), we obtain these possible starting formulas: 


$$
\begin{aligned}
& c_{3} \rightarrow w(u) \cdot\left(d_{3}(u, S)+\delta_{1}+\delta_{2}\right) \\
& c_{1} \rightarrow w(u) \cdot\left(d_{1}(u, S)+\delta_{1}\right) \\
& c_{2} \rightarrow w(u) \cdot\left(d_{2}(u, S)+\delta_{2}\right) \\
& c_{0} \rightarrow w(u) \cdot d_{0}(u, S)
\end{aligned}
$$

The starting formula is given by the occurrence of edges $e_{1}$ and $e_{2}$ in shortest path for $\delta_{1}=\delta_{2}=0$.

2. We find out which cases (from $c_{1}, c_{2}, c_{0}$ ) can also occur, by omitting the edges $e_{1}, e_{2}$. Formulas for these cases are similar to the formulas described above.

3. If the case $c_{3}$ occurs for $\delta_{1}=\delta_{2}=0$, then the bounds of $\Delta_{3}$ are

$w(u) \cdot\left(d_{3}(u, S)+\delta_{1}+\delta_{2}\right)=w(u) \cdot\left(d_{1}(u, S)+\delta_{1}\right)$

$w(u) \cdot\left(d_{3}(u, S)+\delta_{1}+\delta_{2}\right)=w(u) \cdot\left(d_{2}(u, S)+\delta_{2}\right)$

$w(u) \cdot\left(d_{3}(u, S)+\delta_{1}+\delta_{2}\right)=w(u) \cdot d_{0}(u, S)$

Hence, the set $\Delta_{3}$ is given by bounds

$$
\begin{gathered}
\delta_{1}, \delta_{2} \geq 0 \\
d_{3}(u, S)+\delta_{2} \leq d_{1}(u, S) \\
d_{3}(u, S)+\delta_{1} \leq d_{2}(u, S) \\
d_{3}(u, S)+\delta_{1}+\delta_{2} \leq d_{0}(u, S)
\end{gathered}
$$

The set $\Delta_{1}$ (when the case $c_{1}$ occurs) is given by inequalities

$$
\begin{aligned}
& \delta_{3}(u, S)+\delta_{2} \geq d_{1}(u, S) \\
& d_{1}(u, S)+\delta_{1} \leq d_{2}(u, S)+\delta_{2} \\
& d_{1}(u, S)+\delta_{1} \leq d_{0}(u, S)
\end{aligned}
$$

Similarly, $\Delta_{2}$ (when the case $c_{2}$ occurs) is given by

$$
\begin{aligned}
\delta_{1}, \delta_{2} & \geq 0 \\
d_{3}(u, S)+\delta_{1} & \geq d_{2}(u, S) \\
d_{1}(u, S)+\delta_{1} & \geq d_{2}(u, S)+\delta_{2} \\
d_{2}(u, S)+\delta_{2} & \geq d_{0}(u, S)
\end{aligned}
$$

The set $\Delta_{0}$ (when the case $c_{0}$ occurs) is given by

$$
\begin{aligned}
\delta_{1}, \delta_{2} & \geq 0 \\
d_{3}(u, S)+\delta_{1}+\delta_{2} & \geq d_{0}(u, S) \\
d_{1}(u, S)+\delta_{1} & \geq d_{0}(u, S) \\
d_{2}(u, S)+\delta_{2} & \geq d_{0}(u, S)
\end{aligned}
$$

4. If the case $c_{1}$ is the starting case, then the bounds of $\Delta_{1}$ are

$$
\begin{aligned}
\delta_{1}, \delta_{2} & \geq 0 \\
d_{1}(u, S)+\delta_{1} & \leq d_{2}(u, S)+\delta_{2} \\
d_{1}(u, S)+\delta_{1} & \leq d_{0}(u, S)
\end{aligned}
$$

Similarly, $\Delta_{2}$ (when the case $c_{2}$ occurs) is given by

$$
\begin{aligned}
\delta_{1}, \delta_{2} & \geq 0 \\
d_{1}(u, S)+\delta_{1} & \leq d_{2}(u, S)+\delta_{2} \\
d_{2}(u, S)+\delta_{2} & \leq d_{0}(u, S)
\end{aligned}
$$

The bounds of $\Delta_{0}$ (when the case $c_{0}$ occurs) are

$$
\begin{gathered}
\delta_{1}, \delta_{2} \geq 0 \\
d_{1}(u, S)+\delta_{1} \leq d_{0}(u, S) \\
d_{2}(u, S)+\delta_{2} \leq d_{0}(u, S)
\end{gathered}
$$

5. The starting case $c_{2}$ is very similar to the previous starting case.

6. If $c_{0}$ is the starting case, then we have only one set $\Delta_{0}$ given by bounds $\delta_{1}, \delta_{2} \geq 0$.

Bounds for formulas of transportation performance can be obtained from bounds computed for every vertex $u \in U$.

\section{Algorithms for computation of pairs of critical edges}

In this section we introduce a fast algorithm, which finds the set of critical edges in the case where the extensions of travel times do not force us to avoid the critical edges.

We start with finding the pair of critical edges, when the values $\delta_{1}$ and $\delta_{2}$ are small - we do not avoid any edge in the shortest path.

1. Find the shortest paths from $u_{i}$ to $S$ for all $u_{i} \in U=$ $=\left\{u_{1}, u_{2}, \ldots, u_{m}\right\}$. The set of all such paths is denoted by $P=\left\{p_{1}, p_{2}, \ldots, p_{m}\right\}$. (The starting vertices of these paths are from $U$.)

2. For every edge $e$ we have

$$
s(e)=\sum_{i=1}^{m} w\left(u_{i}\right)_{s_{i}}(e), \text { where } s_{i}(e)=\left\{\begin{array}{l}
1, \text { if } e \in p_{i} \\
0, \text { if } e \in p_{i}
\end{array}\right.
$$

The sum is taken over all vertices $u \in U$ such that the edge $e$ is the edge of the shortest path from the set $P$ with the starting point $u$.

3. We order the edges of $G$ by $s(e)$ decreasingly. The first two edges form a critical pair of edges. This is a polynomial algorithm which can be generalised for every $k>2$. Let us suppose that the extensions of times are too large, we need to find the new shortest paths without the critical edges. It is, in general, an NP-hard problem. However, for $k=2$, we can solve the problem by brute force.

\section{Conclusions and further research}

We studied a possible characterization of critical pairs of edges in transportation networks. We suggested the approach which allows us to determine the characteristic function of the time elongation of transportation performance for pairs of affected edges.

Future research in this field will focus on the tests on real transportation networks and study the elongation of transportation performance as a function of $k>2$ variables - it means that we will also consider scenarios with $k>2$ critical edges. 


\section{Acknowledgment}

This work was supported by the research grants VEGA 1/0342/18 "Optimal dimensioning of service systems", VEGA
1/0463/16 "Economically efficient charging infrastructure deployment for electric vehicles in smart cities and communities" and APVV-15-0179 "Reliability of emergency systems on infrastructure with uncertain functionality of critical elements".

\section{References}

[1] GARCIA, S., LABBE, M., MARIN, A.: Solving Large p-median Problems with a Radius Formulation. INFORMS Journal on Computing, 23(4), 546-556, 2011. https://doi.org/10.1287/ijoc.1100.0418

[2] JANACEK, J., KVET, M.: Relevant Network Distances for Approximate Approach to Large p-median Problems. Operations Research Proceedings 2012, selected papers of the International Annual Conference of the German Operations Research Society (GOR), Germany, 123-128, 2012. https://doi.org/10.1007/978-3-319-00795-3_18

[3] KVET, M.: Computational Study of Radial Approach to Public Service System Design with Generalized Utility. Digital Technologies, Proceedings of the $10^{\text {th }}$ International IEEE Conference, Slovakia, 187-197, 2014. https://doi.org/10.1109/DT.2014.6868713

[4] KVET, M., MATIASKO, K.: Concept of Dynamic Index Management in Temporal Approach Using Intelligent Transport Systems. ROCHA, A., CORREIA, A. M., ADELI, H., REIS, L. P., COSTANZO, S. (Eds.): Recent Advances in Information Systems and Technologies, Vol. 1, Springer, p. 549-560, 2017.

[5] JENELIUS, E.: Network Structure and Travel Patterns: Explaining the Geographical Disparities of Road Network Vulnerability. Journal of Transport Geography, 17(3), 234-244, 2009. https://doi.org/10.1016/j.jtrangeo.2008.06.002

[6] SCOTT, D. M., NOVAK, D. C., AUlTMAN-HALL, L., GUO, F.: Network Robustness Index: A New Method for Identifying Critical Links and Evaluating the Performance of Transportation Networks. Journal of Transport Geography, 14(3), 215-227, 2006. https://doi.org/10.1016/j.jtrangeo.2005.10.003

[7] SUlliVAN, J. L., NOVAK, D. C., AUlTMAN-HALL, L., SCOTT, D. M.: Identifying Critical Road Segments and Measuring System-Wide Robustness in Transportation Networks with Isolating Links: A Link Based Capacity Reduction Problem. Transportation Research Part A., 44, 323-336, 2010. https://doi.org/10.1016/j.tra.2010.02.003

[8] JANACEK, J., KVET, M.: Characteristic of a Critical Network Arc in a Service System. Transport Problems, 12, 141-146, 2017. https://doi.org/10.20858/tp.2017.12.se.12 